Self-Interference Mitigation in Full-Duplex Base-Station using Dual Polarized Reflect-Array

Zarifeh, Nidal; Mai, Alissa; Khaliel, Maher; Kaiser, Thomas

This text is provided by DuEPublico, the central repository of the University Duisburg-Essen.

This version of the e-publication may differ from a potential published print or online version.

DOI: https://doi.org/10.23919/GEMIC.2018.8335059

URN: urn:nbn:de:hbz:464-20180525-103419-6

Link: http://duepublico.uni-duisburg-essen.de/servlets/DocumentServlet?id=46184

Legal notice:

(C) 2018 IMA e.V. Published on DuEPublico with kind permission of IMA e.V. Personal use of this material is permitted. Permission from IMA e.V. must be obtained for all other uses.

Source: This is the author's version of an article that has been published in 2018 11th German Microwave Conference (GeMiC) : [Proceedings] - IEEE, 2018 ISBN 978-3-9812668-8-7.

https://doi.org/10.23919/GEMIC.2018.8335059 


\title{
Self-Interference Mitigation in Full-Duplex Base-Station using Dual Polarized Reflect-Array
}

\author{
Nidal Zarifeh, Mai Alissa, Maher Khaliel, Thomas Kaiser \\ Institute of Digital Signal Processing, Duisburg-Essen University, Germany \\ nidal.zarifeh@uni-due.de
}

\begin{abstract}
This paper proposes the use of reflect-array to mitigate self-interference in the propagation domain aiming to achieve a full-duplex mobile system. An ultra-wideband reflectarray is designed to enable full-duplex in an indoor/outdoor LTE base-station with half-duplex users. The antenna design is customized to meet the full-duplex requirements by generating two cross-polarized beams using two separate feeders to achieve high isolation between the downlink and uplink signals. The paper also analyzes the components of self-interference in the direct and back-scattered paths, and the amount of isolation that can be achieved in a wideband system. Both reflect-array and full-duplex technologies are strong candidates to be used in 5G.

Index Terms-reflect-array, full-duplex, self-interference, 5G
\end{abstract}

\section{INTRODUCTION}

The existing generations of mobile communication depend completely on half-duplex (HD) transmission schemes, in which the transmitted and received signals are separated either in time, Time Division Duplexing (TDD), or in frequency domain, Frequency Division Duplexing (FDD). This causes an obvious limitation in resource utilization as these systems invest only half of the available resources. Moreover, most of the traditional approaches for increasing spectral efficiency, such as Adaptive Coding and Modulation (ACM), Multiple Input Multiple Output (MIMO) and smart antennas, have nearly reached their maximum limits [1]. Therefore, there is a major interest in restructuring communication systems to enable In-Band-Full-Duplex (IBFD), or shortly known as (FD). FD communication implies the possibility to double the capacity of wireless communication, and it is considered as a key technology for $5 \mathrm{G}$ systems.

The main challenge facing FD system design is how to suppress the self-interference (SI) signal, i.e. the transmitted signal that leaks from the transmitter into the receiver of the same device. Suppressing such interference is the crucial key issue of FD realization, taking into consideration that the transmitted signal power is about $80-120 \mathrm{~dB}$ higher than the power of the desired received signal from the remote node in most of standard systems [2]. In 5G systems, the trend is mainly towards short-range coverage, where the cell-edge path loss is less than that in traditional cellular systems, making the problem of SI suppression more solvable [1].

Generally, self-interference cancellation (SIC) is implemented in three domains: propagation, analog, and digital domains. None of these domains can meet the required cancellation value per se. Therefore, hybrid solutions are proposed in the literature [1]. In propagation domain, several SIC techniques has been presented to mitigate SI, for example: Antenna conditional placement [3], [4], Directional isolation [5], [6], Absorptive/Reflective Shielding [7], [8], and Crosspolarization [8], [9]. In average these techniques can achieve about $15 \mathrm{~dB}$ of isolation practically. Few works reached almost $70 \mathrm{~dB}$ but in optimal conditions, like using anechoic chamber with narrow band signals. This urges to think about other antenna techniques that can achieve higher SI isolation in practical conditions. Massive MIMO systems have been proposed for $5 \mathrm{G}$ due to their compatibility with small cells. However, Massive MIMO is complex and less adaptable with shorter wavelengths [10]. Meanwhile Reflect-Array (RA) is getting the attention again because it is much simpler, more flexible, and considerably cheaper compared to Massive MIMO. This makes RA a strong candidate technology in some $5 \mathrm{G}$ scenarios [10].

A reflect-array consists of an array of radiating elements that provides an adjusted phasing to form a focused beam when it is illuminated by a feed. Printed RAs combine certain advantages of reflector antennas and phased arrays [11]. Reflect-array can perform like a phased array antenna but without the use of any power divider or additional phase shifters. The simple design of RA and its low power consumption make it economically more applicable in 5G systems [10]. Moreover, its adaptability to high frequencies makes it suitable for high gain and high bandwidth operation. Previously, RA was considered a narrow bandwidth antenna, and this well-known limitation was due to the narrow band nature of the microstrip patches and different spatial phase delays between the feed and each element of the reflect-array [12]. Recently, many works have been done to have better performance of RA in wide/ultra-wide bands (UWB) such as [13]-[16]. The high gain performance can be obtained by increasing the size of the RA which can produce sharp beams, however, this would also increase the different spatial phase delays between the feed and each element of the reflect-array [17].

The novelty of this paper is that it proposes, for the first time, the use of RA to mitigate SI in propagation domain for mobile communication. The UWB RA designed in [13] is considered to enable FD in an indoor/outdoor base-station. The antenna design is customized to meet the FD requirements, where two cross-polarized RAs and two feeders are used to generate DL and UL beams from and to HD users. The paper also analyzes the components of SI in the direct and backscattered paths, and the amount of isolation can be achieved 
using reflect-arrays in a wideband system.

The rest of the paper is organized as follows: In Section II the system is modelled within two parts, the dual-polarized $\mathrm{RA}$, and the equivalent baseband system. Then Section III describes the simulation setup. The results of simulation are presented in Section IV. Finally Section V discusses the advantages and disadvantages of the approach and concludes the paper.

\section{SYSTEM MODEL}

\section{A. Dual Polarized Reflect-Array Antenna}

The low cross-polarization level is essential to isolate the UL and DL beams. Therefore, an UWB orthogonal beams RA antenna is developed. The double ring structure could be utilized as the array cell by cutting gaps across the rings in the cross-polarized direction. Thus, the cross-polarization patterns are cancelled. However, a polarization selective dipole is preferred to increase the isolation level between the copolarized and cross-polarized re-radiated fields.

The basic cell is described in Fig. 1.a, where the 360 degree phase response is acquired using two closed resonant dipoles. The orthogonal polarization is produced by using these two dipoles in a vertical arrangement for the co-polarization beam and in a horizontal arrangement for the cross-polarization beam. Therefore, the best cell dimensions and substrate parameters for one polarization are first calculated and optimized for bandwidth enlargement. After that, these lengths are calculated to produce the cross-polarized beams at two different directions to minimize the cross-polarization levels.

The cross-polarized RA antenna configuration is described in Fig. 2, where two spatially separated orthogonal feeders are utilized to feed the RA surface and in accordance different beam in each polarization is produced. Thus, the crosspolarization levels are further reduced. The relative dipole lengths are calculated to produce an offset beam directed at, for example, $\left(\theta=30^{\circ}, \phi=0^{\circ}\right)$ for the vertical polarization and the other horizontally polarized beam is directed at $\left(\theta=-30^{\circ}\right.$, $\phi=0^{\circ}$ ), where $\theta$ and $\phi$ are the azimuth and the elevation angles respectively.
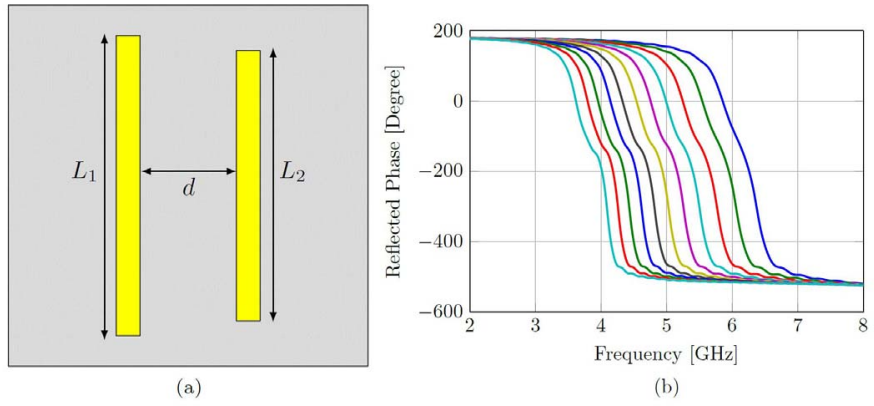

Fig. 1: UWB RA cross-polarized cell: (a) Cell shape consists of two coupled dipoles with relative lengths $L_{2}=0.9 L_{1}$. (b) Reflected phase with the frequency at different lengths of the first dipole.

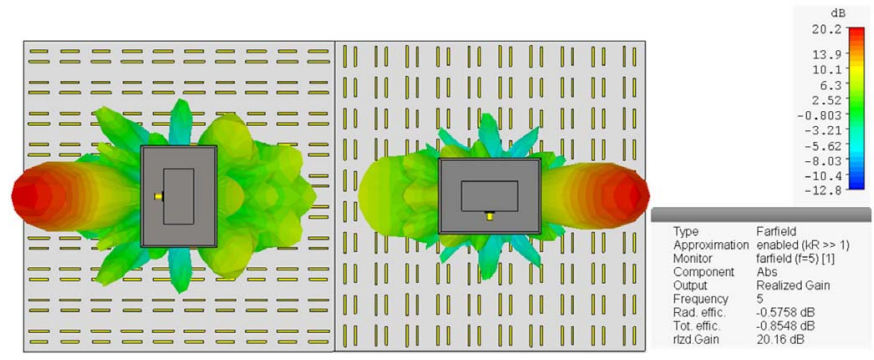

Fig. 2: The radiation patterns of the cross-polarized reflectarrays.

\section{B. The Baseband Model}

The received signal at the base-station can be modelled as:

$$
\mathbf{Y}_{U L}=\sum_{i=1}^{U} \mathbf{H}_{u, i} \mathbf{X}_{u, i}+\mathbf{H}_{S I, d} \mathbf{X}_{D L}+\sum_{j=1}^{D} \mathbf{H}_{S I, s} \mathbf{X}_{d, j}+\mathbf{W}
$$

where $\mathbf{X}_{u, i}$ is the desired signal from the user $i$ and $\mathbf{H}_{u, i}$ is the related UL channel. $\mathbf{X}_{d, j}$ is the DL signal to the user $j$, meanwhile $\mathbf{X}_{D L}$ is the sum of all DL signals. $\mathbf{H}_{S I, d}$ is the direct SI channel, and $\mathbf{H}_{S I, s}$ is the back-scattered SI channel. $U$ and $D$ are the numbers of uplink and downlink users respectively. $\mathbf{W}$ is the white Gaussian noise. For the simplified case studied in this paper, one user for each direction, the received signal at the base-station from the UL user (TxUE1) is:

$$
\mathbf{Y}_{U L}=\mathbf{H}_{u, 1} \mathbf{X}_{u, 1}+\left(\mathbf{H}_{S I, d}+\mathbf{H}_{S I, s}\right) \mathbf{X}_{d, 1}+\mathbf{W}
$$

Meanwhile the received signal at the DL user (RxUE2) is:

$$
\mathbf{Y}_{d, 2}=\mathbf{H}_{d, 2} \mathbf{X}_{d, 2}+\mathbf{H}_{I U I} \mathbf{X}_{u, 1}+\mathbf{W}
$$

where $\mathbf{H}_{I U I}$ is the channel from UL user (TxUE1) to the DL user (RxUE2) that carries the interference.

\section{Simulation Setup}

Computer Simulation Technology (CST) microwave studio is used to design the UWB RA and generate the radiation patterns. The considered base-station scenarios are carried out using Wireless Insite at $5 \mathrm{GHz}$, then the DL, UL, and SI channels coefficients are exported to MATLAB for the baseband processing. Fig. 3 shows the simulated environments in (a) indoor as a multi-room office, and in (b) outdoor as an urban area. This simulation is confined to one user in each direction with different distances of the two users and omnidirectional antennas.

The baseband system is a Long Term Evolution (LTE) link which is based on QPSK OFDM with 5/10/20 MHz bandwidth and sampled by $30.72 \mathrm{MHz}$. The transmitted power from each transmitter is $0 \mathrm{dBm}$.

\section{RESULTS}

The simulation results of the antenna design are presented in Fig. 4, where the cross-polarized patterns are defined based on Ludwigs third definition [18]. These results at $\theta= \pm 30^{\circ}$ indicate that the cross-polarization level is less than $40 \mathrm{~dB}$. 


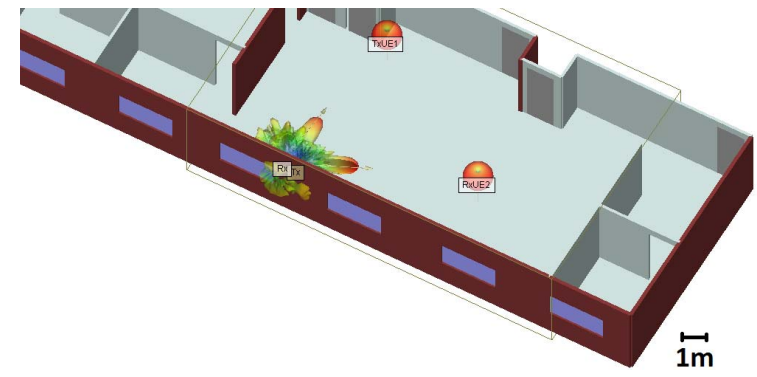

(a)

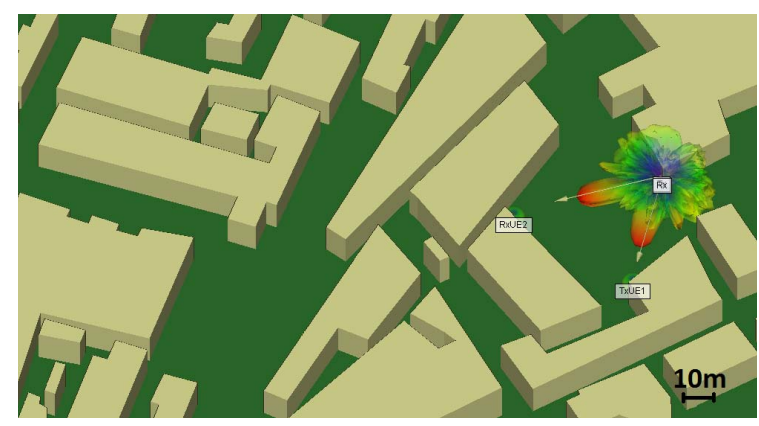

(b)

Fig. 3: The environment of simulation: (a) Indoor (b) Outdoor.

The Side Lobe Level (SLL) is less than $-10 \mathrm{~dB}$, and the RA antenna gain is about $20 \mathrm{~dB}$ which is 4 times higher than the feeder gain. Furthermore, the bandwidth of operation is about $1 \mathrm{GHz}$ for both polarizations. The asymmetry in horizontal and vertical polarization patterns is produced because of feeder and cells asymmetry.

Table I shows the average received power of the four signals in line-of-sight propagation and the components of SI. The first column shows equal values of the DL received power at the DL user (RxUE2), and the UL received power from the UL user (TxUE1). The next four columns show respectively the total power of SI, the signal to self-interference ratio (SSIR), the direct SI, and the strongest scattered path of SI. Finally the IUI is in the last column. It can be noticed that:

The received downlink and uplink signals are weaker in the outdoor due to the longer paths. The path-loss is about $20 \mathrm{~dB}$ more. The average distance at indoor is about $3 \mathrm{~m}$ and at outdoor is $30 \mathrm{~m}$.

The direct SI, caused by the side lobes, is fixed in both environments. The cross-polarization provides $103 \mathrm{~dB}$ mitigation of the direct SI meanwhile it is only $50 \mathrm{~dB}$ with co-polarized arrays.

Also the use of cross-polarization provides higher mitigation of IUI (from TxUE1 to RxUE2). The mitigation enhancement in cross-polarization compared to co-polarization is $-62-(-101)=39 \mathrm{~dB}$ in the indoor and $34 \mathrm{~dB}$ in the outdoor.

The rich scattering environment in the indoor causes higher scattered SI compared to the outdoor.

The FD system cannot perform without the crosspolarization as SSIR is very low with co-polarization.

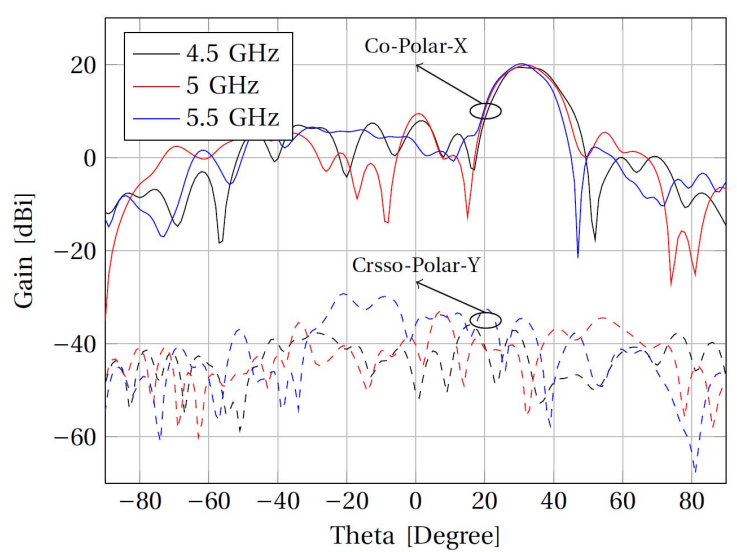

(a)

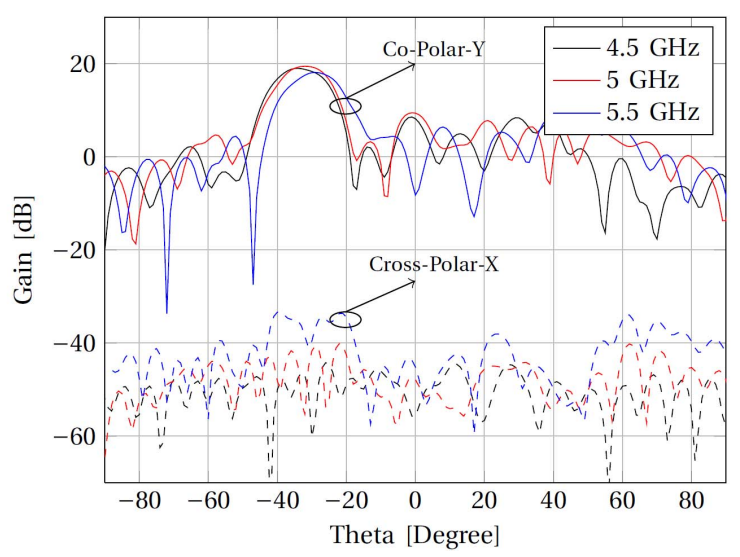

(b)

Fig. 4: The simulated polarization radiation patterns of the dual-polarized RA antenna illustrating the UWB operation: (a) horizontal (b) vertical.

TABLE I: The average power of the four received signals and the signal to self-interference ratio.

\begin{tabular}{|c|c|c|c|c|c|c|}
\hline $\begin{array}{l}\theta= \pm 30^{\circ} \\
P_{T}=0 \mathrm{dBm}\end{array}$ & $\begin{array}{c}\text { UL } \\
\& \text { DL } \\
(\mathrm{dBm})\end{array}$ & $\begin{array}{c}\text { Total } \\
\text { SI } \\
(\mathbf{d B m})\end{array}$ & $\begin{array}{l}\text { SSIR } \\
\text { (dB) }\end{array}$ & $\begin{array}{c}\text { SI } \\
\text { direct } \\
(\mathrm{dBm})\end{array}$ & $\begin{array}{l}\text { SI 1st } \\
\text { scatter } \\
(\mathrm{dBm})\end{array}$ & $\begin{array}{c}\text { IUI } \\
(\mathbf{d B m})\end{array}$ \\
\hline LoS & \multicolumn{6}{|c|}{ Indoor } \\
\hline Co-polar & \multirow{2}{*}{-41} & -45 & 4 & -50 & -72 & -62 \\
\hline X-polar & & -94 & 53 & -103 & -89 & -101 \\
\hline & \multicolumn{6}{|c|}{ Outdoor } \\
\hline Co-polar & \multirow{2}{*}{-63} & -49 & -14 & -50 & -86 & -78 \\
\hline X-polar & & -101 & 38 & -103 & -110 & -112 \\
\hline
\end{tabular}

The average isolation ratio of SI achieved by crosspolarization is $53 \mathrm{~dB}$ at the indoor, and $38 \mathrm{~dB}$ at the outdoor Such values could be achieved with two horn antennas instead of the reflect-arrays, however, the later allow to do beamforming electronically and that cannot be performed by horn antenna without a complicated and impractical mechanical system. Finally, the two remaining figures evaluate the use of the proposed antenna design with an LTE system. Fig. 5 shows that the bit error rate (BER) of the FD system is almost the same as it is without SI in the HD system. However, a slight loss of performance occurs at higher signal to noise ratio 
(SNR) where the self-interference becomes dominant instead of the white noise.

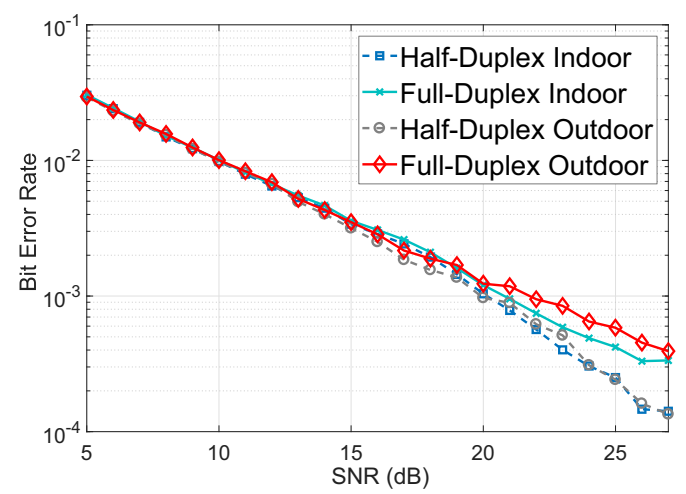

Fig. 5: Bit error rate of FD and HD OFDM systems using cross-polarized RA.

Fig. 6 shows the sum rate of the system. The enhancement of the system capacity is a little less than a double due to residual SI that may be cancelled by traditional SIC digital schemes. Such enhancement is expected to be lower when realizing the setup due to the hardware impairments.

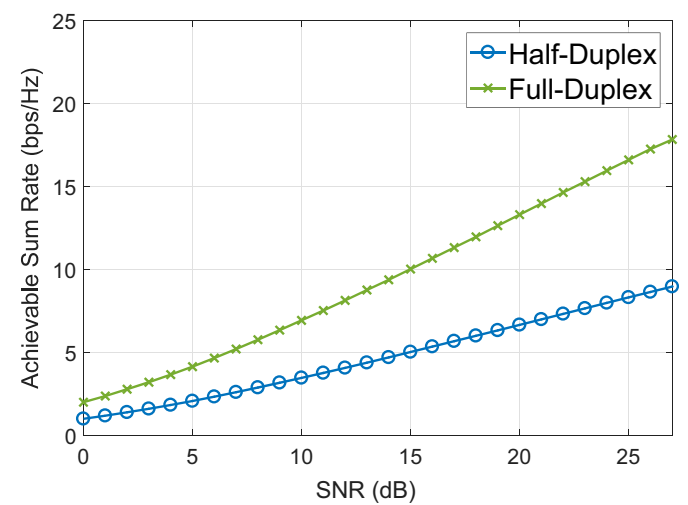

Fig. 6: Achievable sum rate of FD and HD OFDM systems using cross-polarized RA.

\section{CONCLUSION}

This paper verified the benefit of using reflect-array in FD mobile systems. Dual-polarized reflect-arrays were designed to be used in the simulation of a wideband full-duplex basestation with two HD users. The self-interference components were analyzed in indoor and outdoor environments. The system performance was evaluated by studying the BER and the sum rate in a simple case of two users and $60^{\circ}$ of separation. The achievable mitigation in this case with cross-polarized arrays was between 38 and $53 \mathrm{~dB}$ which allowed increasing the total sum rate. As the simulation was confined to a simple case, further work can be done like increasing the number of users and performing the beam-forming for different users' positions. Also the system performance can be enhanced by applying digital cancellation schemes to remove the residual self-interference.

\section{ACKNOWLEDGMENT}

This work was conducted within the framework of the 5G-PPP COHERENT project, which is partially funded by the Commission of the European Union (Grant Agreement No.671639).

\section{REFERENCES}

[1] Kaiser, T. and Zarifeh, N., (2016) General Principles and Basic Algorithms for Full-duplex Transmission, in Signal Processing for 5G: Algorithms and Implementations (eds F.-L. Luo and C. Zhang), John Wiley Sons, Ltd, Chichester, UK. doi: 10.1002/9781119116493.ch16.

[2] Askar, R., Zarifeh, N., Schubert, B., Keusgen, W., and Kaiser, T. (2014) I/Q imbalance calibration for higher self-interference cancellation levels in full-duplex wireless transceivers, in 1st International Conference on 5G for Ubiquitous Connectivity (5GU), 2014, pp. 9297, doi: 10.4108/icst.5gu.2014.258148.

[3] Duarte, M. and Sabharwal, A. (2010) Full-duplex wireless communications using off-the-shelf radios: Feasibility and first results, in Signals, Systems and Computers (ASILOMAR), 2010 Conference Record of the Forty Fourth Asilomar Conference on,pp. 15581562.

[4] Choi, J.I., Hong, S., Jain, M., Katti, S.,Levis, P., and Mehlman, J. (2012) Beyond full duplex wireless, in Signals, Systems and Computers (ASILOMAR), 2012 Conference Record of the Forty Sixth Asilomar Conference on, pp. 4044, doi:10.1109/ACSSC.2012.6488954.

[5] Everett, E., Sahai, A., and Sabharwal, A.(2014) Passive SelfInterference Suppression for Full-Duplex Infrastructure Nodes. Wireless Communications, IEEE Transactions on, 13 (2), 680694, doi:10.1109/TWC.2013.010214.130226.

[6] Duarte, M., Sabharwal, A., Aggarwal, V.,Jana, R., Ramakrishnan, K., Rice, C., and Shankaranarayanan, N. (2014) Design and Characterization of a Full-Duplex Multiantenna System for WiFi Networks. Vehicular Technology, IEEE Transactions on, 63 (3), 11601177,doi:10.1109/TVT.2013.2284712.

[7] Anderson, C., Krishnamoorthy, S., Ranson,C., Lemon, T., Newhall, W., Kummetz, T., and Reed, J. (2004) Antenna Isolation,Wideband Multipath Propagation Measurements, and Interference Mitigation for On-frequency Repeaters, in SoutheastCon, 2004. Proceedings. IEEE, pp. 110114, doi: 10.1109/SECON.2004.1287906.

[8] Everett, E., Sahai, A., and Sabharwal, A.(2014) Passive SelfInterference Suppression for Full-Duplex Infrastructure Nodes. Wireless Communications, IEEE Transactions on, 13 (2), 680694 , doi:10.1109/TWC.2013.010214.130226.

[9] Khandani, A. (2010), Methods for spatial multiplexing of wireless two-way channels.URL http://www.google.com/patents/US7817641, uS Patent 7,817,641.

[10] M. H. Dahri, M. H. Jamaluddin, M. I. Abbasi and M. R. Kamarudin, "A Review of Wideband Reflectarray Antennas for 5G Communication Systems," in IEEE Access, vol. 5, pp. 17803-17815, 2017. doi: 10.1109/ACCESS.2017.2747844

[11] J. Huang and J. A. Encinar, Reflectarray antennas. Piscataway, NJ/New York: IEEE Press/Wiley, 2008.

[12] J. Huang, Bandwidth study of microstrip reflectarray and a novel phased reflectarray concept, in Proc. IEEE AP-S/URSI Symp., Newport Beach, CA, Jun. 1995, pp. 582585.

[13] M. Khaliel, A. Fawky, M. El-Hadidy, T. Kaiser, "UWB Reflectarray Antenna for Chipless RFID Applications," 2014 31st National Radio Science Conf., Cairo Egypt, pp.17-20, 28-30 Apr. 2014.

[14] M. Abd-Elhady, S. Zainud-Deen, A. Mitkees, and A. Kishk, Wideband Rectangular Dielectric Resonator Elements Reflectarray, in Antennas and Propagation (MECAP), 2012 Middle East Conference on, 2012.

[15] M. R. Chaharmir, J. Shaker, N. Gagnon and D. Lee, ”Design of Broadband, Single Layer Dual-Band Large Reflectarray Using Multi Open Loop Elements," in IEEE Transactions on Antennas and Propagation, vol. 58, no. 9, pp. 2875-2883, Sept. 2010.

[16] E. Carrasco, J. A. Encinar and M. Barba, "Bandwidth Improvement in Large Reflectarrays by Using True-Time Delay," in IEEE Transactions on Antennas and Propagation, vol. 56, no. 8, pp. 2496-2503, Aug. 2008.

[17] D. M. Pozar, Bandwidth of reflectarray, Electron. Lett., vol. 39, no. 21, pp. 14901491, Oct. 2003

[18] A. Ludwig, "The definition of cross polarization," in IEEE Transactions on Antennas and Propagation, vol. 21, no. 1, pp. 116-119, Jan 1973. doi: 10.1109/TAP.1973.1140406 